\title{
Role of glycemic variability in gestational diabetes mellitus (GDM): still an uphill climb
}

\author{
Annunziata Lapolla $\cdot$ Nino Cristiano Chilelli
}

Received: 16 September 2012/ Accepted: 18 September 2012/Published online: 28 September 2012

(C) Springer Science+Business Media New York 2012

Insulin resistance tends to increase in physiological pregnancy because of the gradually rising levels of feto-placental hormones such as progesterone, cortisol, growth hormone, prolactin, and human placental lactogen. In response to this imbalance, the pancreas normally compensates with a higher insulin secretion. When this compensatory mechanism fails, glucose intolerance develops and, in pregnancy, this is called gestational diabetes mellitus (GDM) [1]. The resulting maternal hyperglycemia gives rise to a concomitant fetal hyperinsulinemia via the placenta, leading primarily to fetal macrosomia, which is the main cause of well-known complications relating to the fetus's development (e.g., shoulder dystocia, Erb's paralysis, hypoxia, and acidosis) and to the course of labor (e.g., perineal laceration, cesarean section) [2].

Clinical evidence has confirmed that the main goal of glycemic management in GDM is to keep the mother's blood glucose levels as close as possible to the normal range. A number of studies have demonstrated that macrosomia correlate with glycemic control in pregnancy complicated by diabetes [3].

It has recently been suggested that glucose variability, characterized by significant glucose excursions, may overlap with HbAlc levels in determining the risk of diabetes-related complications [4]. Oscillating blood sugar levels have been shown to increase free radicals and endothelial dysfunction, which are the links between hyperglycemia and the activation of pathological pathways that lead to tissue damage [5], and it has been reported that

\footnotetext{
A. Lapolla $(\bowtie) \cdot$ N. C. Chilelli

Department of Medicine (DIMED), University of Padua,

Via Giustiniani n 2, 35128 Padua, Italy

e-mail: annunziata.lapolla@unipd.it
}

containing these hyperglycemic spikes coincides with a reduction in some markers of oxidative stress.

In pregnancy complicated by diabetes even transient periods of hyperglycemia may lead to accelerated fetal growth, resulting in macrosomic infants. These peaks are difficult to capture using common glucose monitoring methods based on six to eight glucose measurements a day, whereas continuous glucose monitoring (CGM) systems are more revealing because they enable glucose levels to be monitored constantly throughout the day [6].

Despite recent opportunities to shed further light on the subject, glucose variability is still a poorly understood factor in GDM patients, especially its link with maternal and fetal complications. In one study, 48-h CGM was performed in all three trimesters in 31 women with GDM, and showed a close relationship between babies' ponderal index and their mothers' glucose variability indicators and mean glycemia in the 2nd trimester, irrespective of their $\mathrm{HbA1c}$ levels. This study emphasized that fetal growth in the early stages of gestation could be affected even in patients whose glycemic variability indices were only slightly higher than in controls [7].

In this issue of Endocrine, Su et al. [8] set out to use 72-h CGM to analyze several glycemic variability parameters in 30 patients with GDM, as compared with 20 healthy pregnant women and 20 healthy non-pregnant women, seeking a possible association with markers of beta-cell function. As indicators of glucose variability, they took into account the mean amplitude of glycemic excursion (MAGE), the mean of daily differences (MODD), and the standard deviation of blood glucose (SDBG); the HOMA-IR index was calculated to assess insulin resistance. The early insulinogenic index $\left(\Delta I_{30} / \Delta G_{30}\right)$ and the area under the curve of insulin (AUCI 180) derived from $75 \mathrm{~g}$ oral glucose tolerance test (OGTT) were calculated to 
evaluate early-phase insulin secretion and second-phase insulin secretion, respectively. The authors found a gradual increase in glycemic variability from a healthy condition to a state of glucose intolerance, which is consistent with previous findings [7]. HOMA-IR and AUCI180, two indicators related to hyperinsulinemia, were higher in pregnant women, and in the GDM ones in particular, whereas $\Delta I_{30} /$ $\Delta G_{30}$ was highest in non-pregnant women and lowest in GDM patients. Finally, in a multivariate regression analysis conducted on GDM pregnant women alone, $\Delta I_{30} / \Delta G_{30}$ emerged as the strongest independent contributor to MAGE. This last finding is the really original contribution that comes from this study, on which it is worth adding a few considerations. This is the first study so far to have examined the pathophysiological link between glycemic variability and metabolic abnormalities in patients with GDM. Even considering the small number of cases involved, the importance of the results stems mainly from the use of CGM to monitor the glycemic variability indices. In an earlier study, Kohnert et al. demonstrated a strong association between CGM-derived MAGE and postprandial beta-cell function in a cohort of type 2 diabetic patients not treated with insulin, suggesting a role of impaired early-phase insulin secretion in causing hyperglycemic spikes [9]: Su et al. have confirmed these observations in GDM patients, though they did not adopt the model based on C-peptide kinetics during a mixed-meal test, which is the gold standard method for measuring betacell function.

Despite these encouraging results, there remain at least two major controversies over this issue. First, given the observational design of these studies, it is hard to say whether glycemic variability relates to beta-cell impairment via a cause-effect relationship. Second, as already demonstrated in some studies [10], moreover, glycemic variability indicators generally correlate closely with mean daily blood glucose levels, a parameter not taken into account by $\mathrm{Su}$ et al., and this makes it a little difficult to ascertain whether any changes in glucose variability indicators are directly attributable to pathophysiological causes, or arise indirectly from changes in mean blood glucose profiles. Therefore, much could be clarified by future interventional studies, using therapeutic strategies designed to selectively overcome the early-phase insulin secretion impairment. While this would be relatively easy to achieve in patients with type 1 or type 2 diabetes (for whom new DPP4 inhibitors or GLP-1 analogs may hold some promise), strategies for reducing glycemic variability in pregnant women with GDM are limited for the time being to reinforcing their nutritional education and possibly to careful insulin titration.

Summing up the above considerations, studying glycemic variability, especially focusing on the pathophysiology, remains a very complex and intriguing challenge. Since recent in vitro and in vivo evidences demonstrate glycemic variability as much a result as a cause of the impairment of beta-cell function, future studies such as that conducted by $\mathrm{Su}$ et al. are of crucial importance, in particular with regard to GDM.

\section{References}

1. A. Lapolla, M.G. Dalfrà, G. Mello et al., Early detection of insulin sensitivity and beta cell function with simple tests indicate future derangements in late pregnancy. J. Clin. Endocrinol. Metab. 93, 876-880 (2008)

2. A. Lapolla, M.G. Dalfrà, D. Fedele, Management of gestational diabetes mellitus. Diabetes Metab. Syndr. Obes. 17, 73-82 (2009)

3. HAPO Study Cooperative Research Group, B.E. Metzger, L.P. Lowe, A.R. Dyer et al., Hyperglycemia and adverse pregnancy outcomes. N. Engl. J. Med. 358, 1991-2002 (2008)

4. L. Nalysnyk, M. Hernandez-Medina, G. Krishnarajah, Glycaemic variability and complications in patients with diabetes mellitus: evidence from a systematic review of the literature. Diabetes Obes. Metab. 12, 288-298 (2010)

5. C.M. Chang, C.J. Hsieh, J.C. Huang, I.C. Huang, Acute and chronic fluctuations in blood glucose levels can increase oxidative stress in type 2 diabetes mellitus. Acta Diabetol. (2012). doi: 10.1007/s00592-012-0398-x

6. L. Jovanovic, Continuous glucose monitoring during pregnancy complicated by gestational diabetes mellitus. Curr. Diabetes Rep. 1, 82-85 (2001)

7. M.G. Dalfrà, G. Sartore, G. Di Cianni, G. Mello, C. Lencioni, S. Ottanelli, J. Sposato, F. Valgimigli, C. Scuffi, M. Scalese, A. Lapolla, Glucose variability in diabetic pregnancy. Diabetes Technol. Ther. 13, 853-859 (2011)

8. J.B. Su, X.Q. Wang, J.F. Chen, G. Wu, Y. Jin, F. Xu, X.H. Wang, Y.T. Liu, Glycemic variability in gestational diabetes mellitus and its association with $\beta$ cell function. Endocrine (2012). doi: 10.1007/s12020-012-9753-5

9. K.D. Kohnert, P. Augstein, E. Zander, P. Heinke, K. Peterson, E.J. Freyse, R. Hovorka, E. Salzsieder, Glycemic variability correlates strongly with postprandial beta-cell dysfunction in a segment of type 2 diabetic patients using oral hypoglycemic agents. Diabetes Care 32, 1058-1062 (2009)

10. G. Sartore, N.C. Chilelli, S. Burlina, P.D. Stefano, F. Piarulli, D. Fedele, A. Mosca, A. Lapolla, The importance of HbA1c and glucose variability in patients with type 1 and type 2 diabetes: outcome of continuous glucose monitoring (CGM). Acta Diabetol. (2012). doi:10.1007/s00592-012-0391-4 